\title{
PHYSICS AT TESLA
}

\author{
G.A.BLAIR \\ Department of Physics, Royal Holloway, University of London, Egham, Surrey. \\ TW20 OEX, UK \\ E-mail: g.blair@rhul.ac.uk
}

\begin{abstract}
The physics at a 500-800 GeV electron positron linear collider, TESLA, is reviewed. The machine parameters that impact directly on the physics are discussed and a few key performance goals for a detector at TESLA are given. Emphasis is placed on precision measurements in the Higgs and top sectors and on extrapolation to high energy scales in the supersymmetric scenario.
\end{abstract}

\section{Introduction}

A high energy linear collider (LC) operating in the energy regime $0.5-\sim 1 \mathrm{TeV}$ will provide a programme of precision measurements and possibly new discoveries in an energy regime matching that of the LHC. Worldwide studies ${ }^{1}$ have been exploring the physics potential of the LC, representing machine designs from Japan (JLC), America (NLC) and Europe (TESLA). A path to multi-TeV machines is also proposed by CERN (CLIC). The TESLA design is well advanced and a Technical Design Report (TDR) ${ }^{2}$ was published in March 2001 that covers the machine ${ }^{3}$, the detector and the physics.

\section{Experimental Environment}

The advantage of a linear collider (LC) over a hadronic one (LHC) centres on the facts that the centre of mass (cms) energy of the colliding particles is tunable (given by energy of the colliding beams) and that the events themselves are much cleaner. The LC has the additional advantage that it can provide polarised beams, which can be used to explore the spin structure of fundamental couplings, to reduce systematic errors in precision measurements, to provide an effective luminosity gain in some channels such as the production of chiral supersymmetric particles (sparticles) and, importantly, to reduce dramatically the backgrounds to some processes from $W$-pair production.

Both the LHC and the LC will be needed to explore fully the TeV scale. Some states that are invisible at the LHC, such as an invisible Higgs, would be both discovered and have their properties measured at the LC. Other states that may be discovered at the LHC will, if their mass is not too high, be studied with high precision at the LC. The benefits apply in both directions;

ll proc: submitted to World Scientific on November 19, 2018 
for instance if supersymmetry (SUSY) is realised in nature then the LHC will almost certainly discover it and will tell the LC where to concentrate its threshold scans. However LHC analyses are limited by the overall scale, set by the lightest SUSY particle (LSP) mass. A precision measurement of the LSP mass at the LC would then feed back directly to the LHC analyses. This type of feedback would clearly be greatly beneficial to both programmes and is a strong argument for concurrent running of the LHC and the LC.

\subsection{Machine Parameters}

TESLA will provide tunable $e^{+} e^{-}$collisions at cms energies ranging from the $Z^{0}$ pole up to about $0.8 \mathrm{TeV}$. The machine offers very high luminosity of $3-5 \times 10^{34} \mathrm{~cm}^{-2} \mathrm{~s}^{-1}$. This performance permits a general analysis to assume a total integrated luminosity of order $500 \mathrm{fb}^{-1}$ and specific high-importance analyses (such as the Higgs self coupling, discussed below) to assume an integrated luminosity of order $1 \mathrm{ab}^{-1}$. Electron polarisation of $\sim 80 \%$ is planned and positron polarisation of up to $\sim 60 \%$ is also envisaged (there will be some trade-off between luminosity and positron polarisation). The polarisation can be measured to a precision of about $0.5 \%$.

Although the nominal beam energies will be known to the $10^{-4}$ level, there are additional effects influencing the energy of individual events. One process, already familiar at LEP and SLC energies, is initial state radiation. An additional effect at the LC is beamstrahlung, where the high fields in the colliding bunches cause the emission of large numbers of photons, some of which will in turn produce $e^{+} e^{-}$pairs. Beamstrahlung results in a further widening of the effective electron/positron energy spectrum at the level of $3.3-4.7 \%$.

The TESLA design proposes head-on collisions of electrons with positrons in one interaction region (IR). A second IR is also envisaged with a finite opening angle between the incoming beams. This second IR could be dedicated to $\gamma \gamma$ collisions and would need a specialised detector and final focus layout. In addition to the $e^{+} e^{-}$and $\gamma \gamma$ options, it would also be possible to run in $e^{-} e^{-}$or $e^{-} \gamma$ modes.

\subsection{A Detector for TESLA}

The detector at TESLA must be able to reconstruct high multiplicity multi-jet events as well as be able to measure precisely high-energy single particles. The detector must be hermetic and the TDR design allows energy measurements down to about $27 \mathrm{mrad}$ with tagging of high energy electrons/positrons down to about $4 \mathrm{mrad}$. The low angle region is important for a range of physics

ll proc: submitted to World Scientific on November 19, 2018 
processes; these include multi-jet events where at least one jet will be in the forward direction, SUSY processes - especially when the mass differences between the sparticles are small, and also for measuring (or vetoing) the large numbers of $\gamma \gamma$ events. Tracking at low angles is also important to be able to measure the centre of mass energy spectrum using acolinear Bhabha events. The detector design at low angles, and in the vertex detector region, is constrained by the presence of large numbers of $e^{+} e^{-}$pairs produced by the beamstrahlung photons mentioned in Sec. 2.1. A higher magnetic field is important to contain these particles at low-angle trajectories and a $4 \mathrm{~T}$ detector field is presently being considered.

In a typical hadronic event, the individual particle energies are typically of order $1-2 \mathrm{GeV}$, so the quality of reconstruction hinges on the ability to measure very precisely the positions, directions and energies of lower-energy particles. The process whereby individual particles are reconstructed by combining information optimally from both tracking and calorimetry is called energy flow and the goal performance is set at $\delta E \sim 0.3 \sqrt{E(\mathrm{GeV})}$, a factor of two improvement on that achieved at LEP/SLC. This performance will require very high resolution and high granularity calorimetry, coupled with very high performance tracking.

In addition to measuring well the lower momentum tracks, it is also important to be able to make an excellent measurement of high momentum tracks, for instance to reconstruct the Higgs mass from recoil against the $Z^{0}$ boson (see Sec. 3.1). A global tracking performance of $\delta\left(1 / p_{T}\right) \sim 5 \times 10^{-5} \mathrm{GeVc}^{-1}$ is required. Excellent vertexing, for flavour identification, is essential to a wide range of physics processes, including measuring the branching ratios of the Higgs boson(s) and reconstructing the complicated cascade decay chains of SUSY. A high performance vertex detector is also essential to help with pattern recognition in high-density jets and to provide additional high-precision spatial measurements, which are essential to the global tracking performance.

\section{Physics Processes}

The physics programme at the LC is very rich; a comprehensive overview cannot be given here and only a few highlights from the Higgs and SUSY studies are presented in the following sections. A whole set of interesting measurements can also be made in the top sector, for instance the top quark mass can be measured to a precision where theoretical errors dominate (at $\left.\delta m_{t} \sim 100 \mathrm{MeV}\right)$. Constraining the strong coupling $\alpha_{s}$ to the world average would give a top mass precision of order $40 \mathrm{MeV}$, an order of magnitude improvement on the LHC value ${ }^{4}$. High luminosity running at lower energies

ll proc: submitted to World Scientific on November 19, 2018 
Table 1. Precisions on electroweak parameters from running TESLA at lower energies.

\begin{tabular}{|c|c|c|}
\hline & LEP/SLC/Tevatron & TESLA \\
\hline $\sin ^{2} \theta_{\text {eff }}^{\ell}$ & $0.23146 \pm 0.00017$ & \pm 0.000013 \\
\hline \multicolumn{3}{|c|}{ lineshape observables: } \\
\hline$M_{Z}$ & $91.1875 \pm 0.0021 \mathrm{GeV}$ & $\pm 0.0021 \mathrm{GeV}$ \\
$\alpha_{s}\left(M_{Z^{2}}\right)$ & $0.1183 \pm 0.0027$ & \pm 0.0009 \\
$\Delta \rho_{\ell}$ & $(0.55 \pm 0.10) \cdot 10^{-2}$ & $\pm 0.05 \cdot 10^{-2}$ \\
$N_{\nu}$ & $2.984 \pm 0.008$ & \pm 0.004 \\
\hline heavy flavours: \\
\hline $\mathcal{A}_{b}$ & $0.898 \pm 0.015$ & \pm 0.001 \\
$R_{b}^{0}$ & $0.21653 \pm 0.00069$ & \pm 0.00014 \\
\hline$M_{W}$ & $80.436 \pm 0.036 \mathrm{GeV}$ & $\pm 0.006 \mathrm{GeV}$ \\
\hline
\end{tabular}

(the $Z^{0}$ pole and the $W$-pair threshold) can lead to typically an order of magnitude improvement on the current measurements of electroweak parameters, as shown in Tab. $1^{2}$.

\subsection{Higgs Physics}

Understanding electroweak symmetry breaking lies at the heart of the LC physics programme. This means proving that the Higgs boson has spin-0 and has couplings to the standard model fermions that are proportional to the mass of the fermion, that the couplings to the gauge bosons are those expected from the standard model and that the Higgs potential has the right form, as given by the value of its self couplings.

The Higgs boson can be extensively studied at the LC. Its mass can be measured to high precision, for example a $120 \mathrm{GeV}$ standard model Higgs mass can be measured to $40 \mathrm{MeV}$ by combining all channels and to $70 \mathrm{MeV}$ from using the $Z^{0}$-recoil alone, a method that will work even if the Higgs decays invisibly. In addition, the Higgs tri-linear self coupling can be measured to a statistical precision of about $22 \%$ if its mass is $120 \mathrm{GeV}^{6}$.

The Higgs spin, parity and charge conjugation quantum numbers can also be measured model-independently as can its branching ratios, for which the prospects are shown in Fig. $1^{5}$.

Additional information about the Higgs can also be obtained from increased precision measurements at the LC of the electroweak parameters, such as those listed in Tab. 1. For example, from such precision measurements the Higgs mass can indirectly be constrained at the level of $5 \%{ }^{7}$. Comparing this

ll proc: submitted to World Scientific on November 19, 2018 


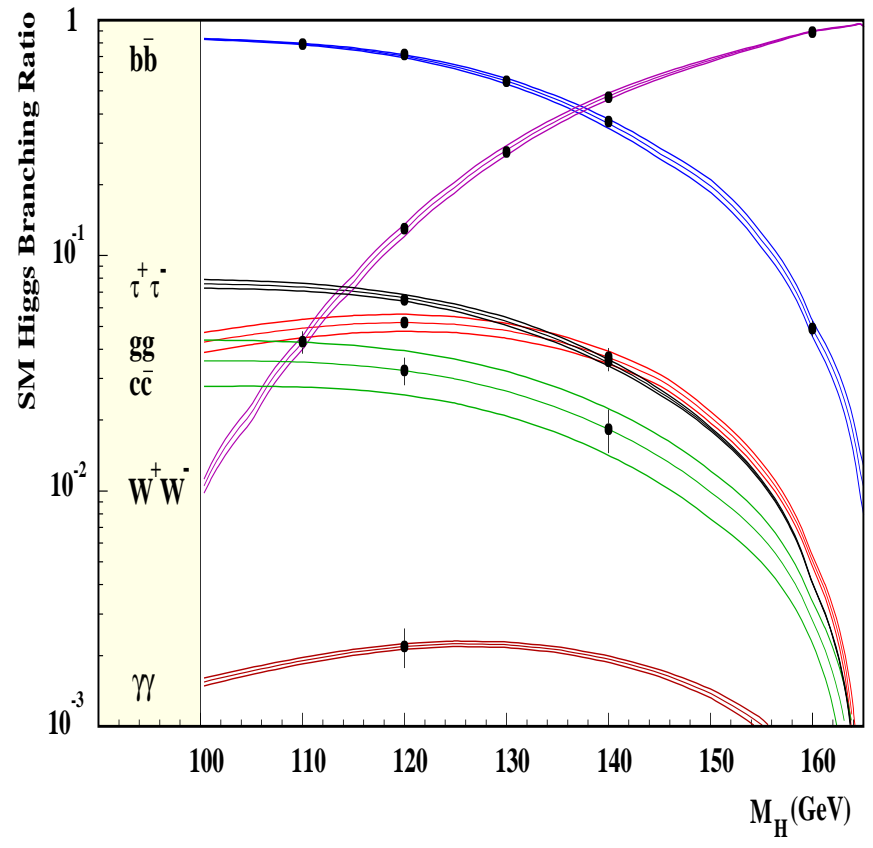

Figure 1. The precisions that can be obtained on the Higgs branching ratios for a range of Higgs masses. The points are the prospective experimental errors, the lines are the standard model uncertainties.

result to that obtained from direct measurements will provide a very powerful test of the Higgs sector.

\subsection{Supersymmetry}

If SUSY is realised in nature, discovering it will require more than simply observing events with missing energy/momentum that are consistent with a given scenario. It will require measuring the couplings of the sparticles, testing that they are the same as those of the particles and also that the sparticles have the correct spin.

The current data favour a light Higgs boson and SUSY provides a mechanism that can accommodate such a light Higgs in a natural manner. The prospects for SUSY thus look good at present and, if SUSY is indeed realised in 
nature, the LC is ideal for making precision measurements of sparticle masses and cross sections. This is achieved by measuring the end-points of the energy spectra of the daughter particles and by performing threshold scans. Using such methods and allowing $100 \mathrm{fb}^{-1}$ per threshold leads typically to per-mille precision on the sparticle masses ${ }^{8}$.

After measuring the masses at the $\mathrm{TeV}$ scale, it is then possible to extrapolate the theory to higher energies by running the renormalisation group equations. In this way, the underlying structure of nature is reconstructed in a model-independent way ${ }^{9}$. Applying this procedure to a minimal supergravity model leads to Fig. 2 where the very fine lines are obtained from the LC measurements of the colourless sparticles and the broader ones assume LHC precisions for the coloured states (which tend to be heavier). In this case, the observation that the curves meet at a common point would indicate that gravity plays a direct role in the breaking of SUSY.

\section{Summary}

The physics and detector studies reported here are only a small subset of a large amount of work by many people. More details can be found in the TDR ${ }^{2}$ and in a complete set of LC-Notes ${ }^{10}$, including work from all the international regions. It is clear that the LC presents a very exciting physics potential and much benefit to both the LHC and the LC programmes could be obtained from a significant time overlap in the running of these machines. The nature of electroweak symmetry breaking, the precision measurement of (s)particle properties and detailed extrapolations to high energy scales may yield model-independent answers to very fundamental questions.

\section{Acknowledgments}

Financial support from DESY and from the British Council ARC programme is gratefully acknowledged.

\section{References}

1. The central web page of the Worldwide Study of the Physics and Detectors for Future Linear $e^{+} e^{-}$Colliders: http : //lcwws.physics.yale.edu/lc/

2. The TESLA Technical Design Report, March 2001. http : //tesla.desy.de/new_pages/TDR_CD/start.html

3. W.Decking, "The TESLA Linear Collider Design", these proceedings.

ll proc: submitted to World Scientific on November 19, 2018 

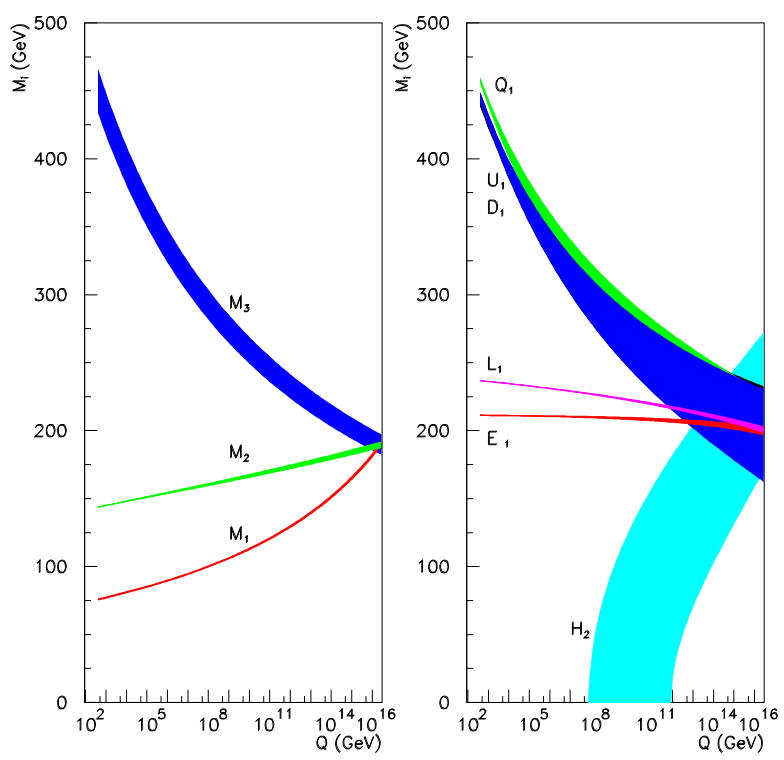

Figure 2. Model-independent extrapolations to high energies of SUSY soft breaking terms. The left hand plot shows the extrapolations of the gaugino mass parameters and the right hand plot the scalar mass parameters. The underlying model is minimal supergravity with $\tan \beta=30, M_{0}=200 \mathrm{GeV}, M_{\frac{1}{2}}=190 \mathrm{GeV}, A_{0}=550 \mathrm{GeV}, \operatorname{sign}(\mu)=-1$.

4. D.Peralta, M.Martinez, R.Miquel Proc. of LCWS99, Sitges (1999).

5. M.Battaglia, Proceedings of LCWS99 Sitges (1999).

6. A.Djouadi, W.Kilian, M.Mühlleitner, P.M.Zerwas EPJC 10, 27 (1999), C.Castanier, P.Gay, P.Lutz, J.Orloff. LC Note LC-PHSM-2000-061.

7. S. Heinemeyer, Th. Mannel, G. Weiglein, Proc. of LCWS99, Sitges (1999). hep-ph/9909538. J. Erler et al. Phys. Lett.B4861252000.

8. U.Martyn, G.A.Blair, Proc. of LCWS99, Sitges (1999). hep-ph/0009186.

9. G.A.Blair, W.Porod, P.M.Zerwas, Phys. Rev. D 63, 17703 (2001).

10. The international LC notes are located at: http : //www.desy.de/ lcnotes/LCnotes_welcome.html. 